\title{
FELL BUNDLES ASSOCIATED TO GROUPOID MORPHISMS
}

\author{
VALENTIN DEACONU, ALEX KUMJIAN and BIRANT RAMAZAN
}

\begin{abstract}
Given a continuous open surjective morphism $\pi: G \rightarrow H$ of étale groupoids with amenable kernel, we construct a Fell bundle $E$ over $H$ and prove that its $C^{*}$-algebra $C_{r}^{*}(E)$ is isomorphic to $C_{r}^{*}(G)$. This is related to results of Fell concerning $C^{*}$-algebraic bundles over groups. The case $H=X$, a locally compact space, was treated earlier by Ramazan. We conclude that $C_{r}^{*}(G)$ is strongly Morita equivalent to a crossed product, the $C^{*}$-algebra of a Fell bundle arising from an action of the groupoid $H$ on a $C^{*}$-bundle over $H^{0}$. We apply the theory to groupoid morphisms obtained from extensions of dynamical systems and from morphisms of directed graphs with the path lifting property. We also prove a structure theorem for abelian Fell bundles.
\end{abstract}

\section{Introduction}

In his Memoir [6], J. M. G. Fell generalizes Mackey's theory of unitary representations of group extensions to a natural enrichment of the concept of Banach *-algebra, called Banach *-algebraic bundle. Given a normal subgroup $K$ of $G$, he constructs a bundle $B$ over $H=G / K$ with the fiber over the neutral element identified with the algebra $L^{1}(K)$, such that $L^{1}(G)$ is isomorphic to the cross-sectional algebra of $B$. He also proves that there is a one-to-one correspondence between isomorphism classes of Banach *-algebraic bundles with one-dimensional fibers over the group $H$ and the family of isomorphism classes of central topological extensions of $H$ by the unit circle T.

Replacing $L^{1}(K)$ by $C^{*}(K)$, we get the notion of $C^{*}$-algebraic bundle over a locally compact group (see [7, §11]). This may be thought of as a continuous version of a group grading in a $C^{*}$-algebra; one may regard the associated $C^{*}$-algebra as a fairly general sort of crossed product of the fiber algebra over the neutral element by the group (in [14] it is shown that the $C^{*}$-algebra is endowed with a coaction by the group).

There is a natural generalization of the notion of $C^{*}$-algebraic bundle to groupoids (see [27]), which when specialized to topological spaces yields the more usual notion of (continuous) $C^{*}$-bundle. Such objects are often called Fell bundles (see [12], [17]). 
The main result of this note is the construction of a Fell bundle associated to certain groupoid homomorphisms. We restrict ourselves to étale groupoids (the range and the source maps are local homeomorphisms), but we expect that our results hold more generally. Given a continuous open surjective morphism $\pi: G \rightarrow H$ of étale groupoids with amenable kernel, we construct a Fell bundle $E$ over $H$ by extending a result of Ramazan's dissertation (see [23]), which appears in [16]. The authors show that if $H$ is a locally compact space, there is a $C^{*}$-bundle over $H$ with fibers given by $C^{*}$-algebras associated to the fibers of $\pi$. It follows easily that $C_{r}^{*}(G)$ is isomorphic to the $C^{*}$-algebra of continuous sections of this bundle. In our situation, their result may be applied to the restriction of $\pi$ to the preimage of $H^{0}$ (which is an étale groupoid) to obtain a $C^{*}$-bundle over $H^{0}$. This forms the "nucleus" of the desired Fell bundle $E$ (that is, its restriction to $H^{0}$ ).

Lee showed in [15, Theorem 4] that if $A$ is a $C^{*}$-algebra and $X$ is a locally compact space, then $A$ may be realized as the $C^{*}$-algebra associated to a $C^{*}$-bundle over $X$ if and only if there is a continuous open surjection $\pi$ : Prim $A \rightarrow X$. In this case the fiber of the bundle over $x \in X$ is the quotient of $A$ corresponding to the closed set $\pi^{-1}(x) \subset \operatorname{Prim} A$. Hence, if $\pi: Y \rightarrow X$ is a continuous open surjection of locally compact spaces, then $C_{0}(Y)$ is realizable as the $C^{*}$-algebra associated to a $C^{*}$-bundle over $X$ with fibers $C_{0}\left(\pi^{-1}(x)\right)$. In Ramazan's result, the space $Y$ is replaced by a groupoid and $\pi$ is required to be a groupoid morphism (where $X$ is regarded as a groupoid). In our result, $X$ is also replaced by a groupoid, but we need Fell bundles rather than $C^{*}$-bundles.

Several examples are considered, coming from extensions of dynamical systems and graph morphisms.

In the last section we consider abelian Fell bundles (the fibers over the unit space are abelian $C^{*}$-algebras) and prove a structure theorem that states that every such bundle arises from a twisted groupoid covering. We also give various examples of abelian Fell bundles and an application of the structure theorem.

\section{Fell bundles over groupoids}

Recall the definition of a Fell bundle over a groupoid $G$ (see [12]). Note that it follows from the first nine axioms that $E_{u}$ is a $C^{*}$-algebra for all $u \in G^{0}$, so it makes sense in (10) to require the positivity of $e^{*} e$ for all $e \in E$.

DEFINITION 2.1. Let $G$ be a locally compact Hausdorff groupoid with unit space $G^{0}$, range and source maps $r, s$ and set of composable pairs $G^{2}$, which admits a left Haar system. A Banach bundle $p: E \rightarrow G$ is said to be a Fell bundle if there is a continuous multiplication $E^{2} \rightarrow E$, where

$$
E^{2}=\left\{\left(e_{1}, e_{2}\right) \in E \times E \mid\left(p\left(e_{1}\right), p\left(e_{2}\right)\right) \in G^{2}\right\},
$$


and an involution $e \mapsto e^{*}$ which satisfy the following axioms ( $E_{g}$ is the fiber $\left.p^{-1}(g)\right)$.

1. $p\left(e_{1} e_{2}\right)=p\left(e_{1}\right) p\left(e_{2}\right) \forall\left(e_{1}, e_{2}\right) \in E^{2}$;

2. the induced map $E_{g_{1}} \times E_{g_{2}} \rightarrow E_{g_{1} g_{2}},\left(e_{1}, e_{2}\right) \mapsto e_{1} e_{2}$ is bilinear $\forall\left(g_{1}, g_{2}\right) \in G^{2}$;

3. $\left(e_{1} e_{2}\right) e_{3}=e_{1}\left(e_{2} e_{3}\right)$ whenever the multiplication is defined;

4. $\left\|e_{1} e_{2}\right\| \leq\left\|e_{1}\right\|\left\|e_{2}\right\| \forall\left(e_{1}, e_{2}\right) \in E^{2}$;

5. $p\left(e^{*}\right)=p(e)^{-1} \forall e \in E$;

6. the induced map $E_{g} \rightarrow E_{g^{-1}}, e \mapsto e^{*}$ is conjugate linear for all $g \in G$;

7. $e^{* *}=e \forall e \in E$;

8. $\left(e_{1} e_{2}\right)^{*}=e_{2}^{*} e_{1}^{*} \forall\left(e_{1}, e_{2}\right) \in E^{2}$;

9. $\left\|e^{*} e\right\|=\|e\|^{2} \forall e \in E$;

10. $e^{*} e \geq 0 \forall e \in E$.

A Fell bundle $E$ is called saturated if $E_{g_{1}} \cdot E_{g_{2}}$ is total in $E_{g_{1} g_{2}}$ for all $\left(g_{1}, g_{2}\right) \in$ $G^{2}$.

2.2. Facts. For $g \in G, E_{s(g)}, E_{r(g)}$ are $C^{*}$-algebras, and $E_{g}$ is a right Hilbert $E_{s(g)}$-module with inner product $\left\langle e_{1}, e_{2}\right\rangle_{s}=e_{1}^{*} e_{2}$ and a left Hilbert $E_{r(g)}{ }^{-}$ module with inner product $\left\langle e_{1}, e_{2}\right\rangle_{r}=e_{1} e_{2}^{*}$. If $E$ is saturated, then $E_{g}$ is an $E_{r(g)}-E_{s(g)}$ equivalence bimodule, and for all $\left(g_{1}, g_{2}\right) \in G^{2}$, multiplication induces an isomorphism $E_{g_{1}} \otimes_{E_{u}} E_{g_{2}} \cong E_{g_{1} g_{2}}$, where $u=s\left(g_{1}\right)=r\left(g_{2}\right)$.

The restriction $E^{0}=\left.E\right|_{G^{0}}$ is a $C^{*}$-algebra bundle, and $C_{0}\left(E^{0}\right)$, the set of continuous sections vanishing at $\infty$, is a $C^{*}$-algebra. We refer to Addendum 2 in [11] for other facts about $C^{*}$-bundles.

Recall that a subset $S$ of a groupoid $G$ is called a bisection if the restrictions of the range and source maps to $S$ are injective.

Lemma 2.3. Let $E$ be a saturated Fell bundle over an étale groupoid $G$ and let $U$ be an open bisection of $G$. Then the completion of $C_{c}(U, E)$, the continuous compactly supported sections on $U$, with respect to the the supremum norm, is an $A-B$ equivalence bimodule, where $A=C_{0}(r(U), E)$ and $B=C_{0}(s(U), E)$, when endowed with the natural inner products and actions.

Proof. The right and left multiplications are given by

$$
(\xi \cdot b)(g)=\xi(g) b(s(g)), \quad(a \cdot \xi)(g)=a(r(g)) \xi(g),
$$

for $a \in A, b \in B, \xi \in C_{c}(U, E), g \in U$. The inner products are

$$
\langle\xi, \eta\rangle_{B}(s(g))=\xi(g)^{*} \eta(g), \quad\langle\xi, \eta\rangle_{A}(r(g))=\xi(g) \eta(g)^{*},
$$


where $\xi, \eta \in C_{c}(U, E), g \in U$. The positivity of the inner products follows from Definition 2.1.10.

For $G$ an étale groupoid and $p: E \rightarrow G$ a Fell bundle, one can define multiplication and involution on the space of compactly supported continuous sections $C_{c}(E)$ by

$$
(\xi \eta)(g)=\sum_{g=g_{1} g_{2}} \xi\left(g_{1}\right) \eta\left(g_{2}\right), \quad \xi^{*}(g)=\xi\left(g^{-1}\right)^{*}
$$

for $\xi, \eta \in C_{c}(E)$. Define an inner product $\langle\xi, \eta\rangle=P\left(\xi^{*} \eta\right)$ for $\xi, \eta \in C_{c}(E)$, where $P: C_{c}(E) \rightarrow C_{c}\left(E^{0}\right)$ is the restriction map; denote by $L^{2}(E)$ the completion of $C_{c}(E)$ in the norm defined by this inner product (so $\|\xi\|^{2}=$ $\|\langle\xi, \xi\rangle\|)$. Observe that $C_{c}(E)$ acts by left multiplication on $L^{2}(E)$.

Definition 2.4. The $C^{*}$-algebra $C_{r}^{*}(E)$ is defined as the completion of $C_{c}(E)$ in $\mathscr{L}\left(L^{2}(E)\right)$, with respect to the operator norm.

REMARK 2.5. The restriction map $C_{c}(E) \rightarrow C_{c}\left(E^{0}\right)$ extends to a faithful conditional expectation $P: C_{r}^{*}(E) \rightarrow C_{0}\left(E^{0}\right)$. Also, $L^{2}(E)$ is the Hilbert module associated to a bundle of Hilbert modules $V$ over $G^{0}$, where $V_{u}=$ $\bigoplus_{s(g)=u} E_{g}$ (see [12, 3.3]). If $E$ is saturated, there is a natural action of the groupoid $G$ on the $C^{*}$-algebra bundle $\mathscr{K}(V)$ with fibers $\mathscr{K}\left(V_{u}\right) \cong V_{u} \otimes V_{u}^{*}$. One can form the semi-direct product bundle $G \ltimes \mathscr{K}(V)$ over $G$. Kumjian proved in $[12,4.5]$ that, if $E$ is saturated, then $C_{r}^{*}(G \ltimes \mathscr{K}(V))$ and $C_{r}^{*}(E)$ are strongly Morita equivalent.

\section{The Fell bundle associated to a groupoid morphism}

Definition 3.1. Let $G$ and $H$ be topological groupoids. A groupoid morphism $\pi: G \rightarrow H$ is a continuous map that intertwines both the range and source maps and that satisfies $\pi\left(g_{1} g_{2}\right)=\pi\left(g_{1}\right) \pi\left(g_{2}\right)$ for all $\left(g_{1}, g_{2}\right) \in G^{2}$. It follows that $\pi\left(G^{0}\right) \subset H^{0}$.

DEFINITION 3.2. A groupoid fibration is an open surjective morphism of locally compact groupoids $\pi: G \rightarrow H$ with the property that for any $h \in H$ and $x \in G^{0}$ with $\pi(x)=s(h)$ there is $g \in G$ with $s(g)=x$ and $\pi(g)=h$. If $g$ is unique for any such $h$ and $x$, then $\pi$ is called a groupoid covering. Note that for a groupoid covering we have $\pi^{-1}\left(H^{0}\right)=G^{0}$ (see [4]).

For example, if $Y \rightarrow X$ is a Serre fibration of topological spaces, then $\pi_{1}(Y) \rightarrow \pi_{1}(X)$ is a groupoid fibration, and if $\tilde{X} \rightarrow X$ is a covering, then $\pi_{1}(\tilde{X}) \rightarrow \pi_{1}(X)$ is a groupoid covering. Here $\pi_{1}(X)$ denotes the fundamental groupoid of the space $X$.

The following is essentially a restatement of Ramazan's result. 
Lemma 3.3. Given an open surjective morphism $\pi: K \rightarrow X$, where $K$ is a locally compact amenable groupoid and $X$ is a locally compact space, there is a $C^{*}$-bundle $F$ over $X$ with fibers $C_{r}^{*}(K(x))$, where $K(x)=\pi^{-1}(x)$. Moreover, $C_{r}^{*}(K)$ is isomorphic to the $C^{*}$-algebra of continuous sections of this bundle.

Proof. We take $F$ to be the disjoint union of $C_{r}^{*}(K(x))$ over $x \in X$ with the bundle structure defined as in Proposition 1.6 in [6] with $\Gamma=C_{c}(K)$; we may view $\Gamma$ as sections of this bundle by means of the canonical maps $\pi_{x}$ : $C_{c}(K) \rightarrow C_{r}^{*}(K(x))$. The continuity of the norm is proved in Théorème 2.4.6 in Ramazan's thesis ([23]) or Corollary 5.6 in [16]. Using Proposition 1.7 in [6] with $\Gamma$ the compactly supported sections of $F$, we get that $C_{r}^{*}(K) \cong C_{0}(F)$.

THEOREM 3.4. Given an open surjective morphism of étale groupoids $\pi$ : $G \rightarrow H$ with amenable kernel $K:=\pi^{-1}\left(H^{0}\right)$, there is a Fell bundle $E=$ $E(\pi)$ over $H$ such that $C_{r}^{*}(G) \cong C_{r}^{*}(E)$. Moreover, $C_{r}^{*}(G)$ is strongly Morita equivalent to a crossed product $C_{r}^{*}(H \ltimes \mathscr{K}(V))$ (see Remark 2.5).

Proof. Using the lemma for the restriction of $\pi$ to $K=\operatorname{ker} \pi=\pi^{-1}\left(H^{0}\right)$, we get a $C^{*}$-bundle $F$ with fibers $C_{r}^{*}(K(x))$ over the unit space $H^{0}$. We will extend this $C^{*}$-algebra bundle to a Fell bundle $E=E(\pi)$ over $H$.

Note that $K$ is an open étale subgroupoid of $G$, and there is a faithful conditional expectation $\Phi: C_{r}^{*}(G) \rightarrow C_{r}^{*}(K)$. Indeed, if $\Psi: C_{r}^{*}(K) \rightarrow$ $C_{0}\left(K^{0}\right)$ is the canonical conditional expectation, where $K^{0}=G^{0}$ is the unit space, then $\Phi$ must be faithful since $\Psi \circ \Phi$ is faithful. We construct a Hilbert module $M(\Phi)$ over $C_{r}^{*}(K)$ by completing $C_{c}(G)$ with respect to the norm given by the inner product $\left\langle f_{1}, f_{2}\right\rangle=\Phi\left(f_{1}^{*} f_{2}\right)$ for $f_{1}, f_{2} \in C_{c}(G)$. The right multiplication is given by convolution with elements in $C_{c}(K) \subset C_{r}^{*}(K)$ (and extending by continuity). Since $C_{r}^{*}(K) \cong C_{0}(F)$ and $F$ is fibered over $H^{0}$, it follows that $M(\Phi) \cong C_{0}(B)$, where $B$ is a bundle of Hilbert modules over $H^{0}$, with $B_{x}$ a Hilbert module over $F_{x}=C_{r}^{*}(K(x))$ for each $x \in H^{0}$ (see 1.7 in [12]). The fiber $B_{x}$ is the completion of $C_{c}(G(x))$, where $G(x)=\{g \in G \mid$ $s(\pi(g))=x\}$ and the inner product is the natural restriction of the above inner product on $C_{c}(G)$. We define $E_{x}=F_{x}$ for $x \in H^{0}$, and for arbitrary $h \in H$, we define $E_{h}$ to be the completion of $C_{c}\left(\pi^{-1}(h)\right)$ in the norm coming from the inclusion $C_{c}\left(\pi^{-1}(h)\right) \subset C_{c}(G(s(h))) \subset B_{s(h)}$. Note that $E_{h}$ is a submodule of $B_{s(h)}$. The multiplication $E_{h_{1}} \times E_{h_{2}} \rightarrow E_{h_{1} h_{2}}$ is defined by

$(\xi \eta)(g)=\sum_{g_{1} g_{2}=g} \xi\left(g_{1}\right) \eta\left(g_{2}\right), \quad$ for $\quad \xi \in C_{c}\left(\pi^{-1}\left(h_{1}\right)\right), \eta \in C_{c}\left(\pi^{-1}\left(h_{2}\right)\right)$;

for $\xi \in C_{c}\left(\pi^{-1}(h)\right)$ we define $\xi^{*} \in C_{c}\left(\pi^{-1}\left(h^{-1}\right)\right)$ by $\xi^{*}(g)=\overline{\xi\left(g^{-1}\right)}$. Observe that the norm on $E_{h}$ inherited from $B_{s(h)}$ satisfies $\|\xi\|=\left\|\xi^{*} \xi\right\|^{1 / 2}$ for 
$\xi \in E_{h}$. Moreover, the element $\xi^{*} \xi \in E_{s(h)}$ is positive since $\xi^{*} \xi=\langle\xi \text {, } \xi\rangle_{s(h)}$, where $\langle\cdot, \cdot\rangle_{s(h)}$ is the inner product on $B_{s(h)}$. The bundle structure for the union of $E_{h}$ 's is given by $C_{c}(G)$, using the fact that each element in $C_{c}\left(\pi^{-1}(h)\right)$ is the restriction of an element in $C_{c}(G)$ (see Proposition 10.7 in [7]). To prove the continuity of the norm, fix $h_{0} \in H$ and take $U$ an open bisection of $H$ containing $h_{0}$. For $\xi \in C_{c}(G)$, denote by $\xi_{h}$ the restriction of $\xi$ to $\pi^{-1}(h)$. By a partition of unity argument, it will be sufficient to consider $\xi$ with support in $\pi^{-1}(U)$. Since $\left\|\xi_{h}\right\|=\left\|\xi_{h}^{*} \xi_{h}\right\|^{1 / 2}$ and $\xi_{h}^{*} \xi_{h}=\left(\xi^{*} \xi\right)_{s(h)}$, it follows that the map $x \mapsto\left\|\left(\xi^{*} \xi\right)_{x}\right\|$ on $s(U)$ is continuous. It is straightforward to check all the other axioms of a Fell bundle. The bundle $E$ is always saturated.

We can now identify $C_{r}^{*}(K)$ with $C_{0}\left(E^{0}\right)$, where $E^{0}$ is the restriction of $E$ to $H^{0}$. To prove that $C_{r}^{*}(G) \cong C_{r}^{*}(E)$, we use the natural extension of the map $\psi: C_{c}(G) \rightarrow C_{c}(E)$ given by

$$
\psi(f)(h)=\left.f\right|_{\pi^{-1}(h)} \in C_{c}\left(\pi^{-1}(h)\right) \subset E_{h}
$$

to get an isomorphism $U_{\psi}$ between the $C_{0}\left(E^{0}\right)$-Hilbert modules $M(\Phi)$ and $L^{2}(E)$ (see $\$ 2$ before Definition 2.4). Indeed, the module structures and the inner product are preserved since in each case both are derived from convolution and involution on $C_{c}(G)$ (note that $\psi$ is a map of $*$-algebras).

Both $C_{r}^{*}(G)$ and $C_{r}^{*}(E)$ are represented on these isomorphic Hilbert modules, using the left regular representation. The same map $\psi$ preserves the product, and it induces an isomorphism $\alpha_{\psi}=\operatorname{Ad} U_{\psi}$ between these $C^{*}$ algebras.

The last part of the statement follows from Kumjian's result mentioned in Remark 2.5.

EXAmPLe 3.5. Consider $G$ a discrete group, $K$ a normal subgroup and let $H=G / K$ with $\pi: G \rightarrow H$ the canonical morphism. Then we get a Fell bundle $E$ over $H$ with the fiber $C_{r}^{*}(K)$ over the identity element, such that $C_{r}^{*}(G) \cong C_{r}^{*}(E)$. This is a particular case of the construction done by Fell in the context of homogeneous Banach *-algebraic bundles over locally compact groups (see Example 3 on page 77 in [6]). Recall that a Fell bundle over a discrete group is equivalent to a grading.

We specialize to the discrete 3-dimensional Heisenberg group $G \subset$ $S L(3, \mathrm{Z})$. The group $G$ consists of matrices of the form

$$
[a, b, c]:=\left[\begin{array}{ccc}
1 & a & c \\
0 & 1 & b \\
0 & 0 & 1
\end{array}\right] .
$$


The group operation is

$$
[a, b, c]\left[a^{\prime}, b^{\prime}, c^{\prime}\right]=\left[a+a^{\prime}, b+b^{\prime}, c+c^{\prime}+a b^{\prime}\right] .
$$

It is easy to show that $G$ is an extension of $Z^{2}$ by its center $Z$. We have a surjective homomorphism $\pi: G \rightarrow Z^{2}$ given by $\pi[a, b, c]=(a, b)$ with $\operatorname{ker} \pi \cong \mathrm{Z}$. The $C^{*}$-algebra $C^{*}(G) \cong C_{r}^{*}(G)$ is called the rotational algebra in [1], and it may be understood as the algebra of continuous sections of a field of $C^{*}$-algebras over the unit circle T. Our construction from the morphism $\pi$ gives a new perspective: $C^{*}(G)$ is the $C^{*}$-algebra of a Fell bundle over the group $\mathrm{Z}^{2}$ with fibers isometric to $C(\mathrm{~T})$. Note that the complexity of the structure of $C^{*}(G)$ is contained in the definition of the product between the fibers, since for instance $C\left(\mathrm{~T}^{3}\right) \cong C^{*}\left(\mathrm{Z}^{3}\right)$ is also the $C^{*}$-algebra of a Fell bundle over $\mathrm{Z}^{2}$ with fibers isometric to $C(\mathrm{~T})$.

There is another characterization of $G$ as an extension of $\mathbf{Z}$ by $\mathbf{Z}^{2}$ coming from the semi-direct product decomposition $G \cong Z^{2} \rtimes Z$. Here $Z^{2}$ is generated by $[1,0,0]$ and $[0,0,1]$, and the action of $Z$ is defined by conjugation with $[0,1,0]$. The morphism $\pi: G \rightarrow Z$ given by $\pi[a, b, c]=b$ describes $C^{*}(G)$ as the $C^{*}$-algebra of a Fell bundle over $\mathrm{Z}$ with fibers isometric to $C\left(\mathrm{~T}^{2}\right)$.

EXAMPLE 3.6. For $G$ an étale groupoid, consider the equivalence relation

$$
R=\left\{(x, y) \in G^{0} \times G^{0} \mid \exists g \in G \text { such that } r(g)=x, s(g)=y\right\}
$$

and the map $\pi: G \rightarrow R, \pi(g)=(r(g), s(g))$. Assume that the isotropy group bundle is amenable and open in $G$. Then $\pi$ is an open surjective morphism, and $C_{r}^{*}(G)$ may be realized as the $C^{*}$-algebra of a Fell bundle over $R$.

\section{Fell bundles from graph morphisms}

Definition 4.1. Let $V, W$ be (finite) graphs. A graph morphism $\phi: V \rightarrow W$ is a map which preserves the incidences. If $\phi$ is surjective, we say that it has the path lifting property (or that it is a fibration) if for any vertex $v \in V^{0}$ and any edge $b \in W^{1}$ starting at $w=\phi(v)$ there is an edge $a \in V^{1}$ starting at $v$ with $\phi(a)=b$.

Recall (see [13]) that, if $V$ has no sinks, the graph $C^{*}$-algebra $C^{*}(V)$ is the $C^{*}$-algebra of the amenable groupoid

$$
G_{V}=\left\{\left(a, p-q, a^{\prime}\right) \in X_{V} \times \mathbf{Z} \times X_{V} \mid \sigma^{p}(a)=\sigma^{q}\left(a^{\prime}\right)\right\},
$$

where $\sigma\left(a_{1} a_{2} a_{3} \ldots\right)=a_{2} a_{3} a_{4} \cdots$ is the shift map and $X_{V}$ is the space of infinite paths $a_{1} a_{2} a_{3} \cdots$ of concatenated edges in $V^{1}$. 
Proposition 4.2. Assume that $V$ and $W$ have no sinks. A graph morphism $\phi$ with the path lifting property induces a continuous open surjection

$$
\varphi: X_{V} \rightarrow X_{W}, \quad \text { given by } \varphi\left(a_{1} a_{2} a_{3} \cdots\right)=\phi\left(a_{1}\right) \phi\left(a_{2}\right) \phi\left(a_{3}\right) \cdots
$$

between the infinite path spaces, and an open surjective morphism

$$
\pi: G_{V} \rightarrow G_{W}, \quad \text { given by } \pi\left(a, k, a^{\prime}\right)=\left(\varphi(a), k, \varphi\left(a^{\prime}\right)\right)
$$

between the associated groupoids, which is a fibration with kernel

$$
K=\left\{\left(a, 0, a^{\prime}\right) \in G_{V} \mid \varphi(a)=\varphi\left(a^{\prime}\right)\right\} .
$$

Hence, $C_{r}^{*}\left(G_{V}\right)$ is isomorphic to the $C^{*}$-algebra of a Fell bundle over $G_{W}$.

Proof. To show that $\varphi$ is surjective, consider an infinite path $b_{1} b_{2} \cdots \in X_{W}$ beginning at $w_{1} \in W^{0}$. Since $\phi$ is onto, there is $v_{1} \in V^{0}$ with $\phi\left(v_{1}\right)=w_{1}$. By the path lifting property, there is $a_{1} \in V^{1}$ with $\phi\left(a_{1}\right)=b_{1}$. Continuing inductively, it follows that there is $a_{1} a_{2} \cdots \in X_{V}$ such that $\varphi\left(a_{1} a_{2} \cdots\right)=b_{1} b_{2} \cdots$, and therefore $\varphi$ is surjective. Consider a cylinder set $Z \subset X_{V}$ determined by a finite path $a_{1} \cdots a_{n}$, i.e.

$$
Z=\left\{a_{1} \cdots a_{n} x_{1} x_{2} \cdots \in X_{V} \mid x_{1} x_{2} \cdots \in X_{V}\right\} .
$$

Again by the path lifting property, $\varphi(Z)$ is the cylinder set in $X_{W}$ determined by the finite path $\phi\left(a_{1}\right) \cdots \phi\left(a_{n}\right)$. It follows that $\varphi: X_{V} \rightarrow X_{W}$ is continuous and open.

By definition, we have

$$
\begin{aligned}
\pi\left(\left(a, k, a^{\prime}\right)\left(a^{\prime}, l, a^{\prime \prime}\right)\right) & =\pi\left(a, k+l, a^{\prime \prime}\right)=\left(\varphi(a), k+l, \varphi\left(a^{\prime \prime}\right)\right) \\
& =\pi\left(a, k, a^{\prime}\right) \pi\left(a^{\prime}, l, a^{\prime \prime}\right),
\end{aligned}
$$

and $\pi$ intertwines the range and source maps, therefore is a groupoid morphism. Since $\varphi$ is surjective and maps cylinder sets onto cylinder sets, it follows that $\pi$ is surjective, continuous and open. To show that $\pi$ is a groupoid fibration, consider $h=\left(b, k, b^{\prime}\right) \in G_{W}$ and $a^{\prime} \in G_{V}^{0}=X_{V}$ with $\varphi\left(a^{\prime}\right)=s(h)=b^{\prime}$. Since $\varphi$ is onto and intertwines the shift maps, we can find $g=\left(a, k, a^{\prime}\right) \in G_{V}$ with $\pi(g)=h$. Hence $\pi$ is a groupoid fibration. Now $\left(a, k, a^{\prime}\right) \in \operatorname{ker} \pi$ iff $\varphi(a)=\varphi\left(a^{\prime}\right)$ and $k=0$, and the last statement of the proposition follows from Theorem 3.4.

Example 4.3. Consider the graphs $V, W$ with $V^{0}=\{v\}, W^{0}=\{w\}$, $V^{1}=\{a, b, c\}, W^{1}=\{1,2\}$ and $\phi(a)=\phi(b)=1, \phi(c)=2$. Then $\phi$ induces a continuous map $\varphi:\{a, b, c\}^{\mathrm{N}} \rightarrow\{1,2\}^{\mathrm{N}}$ between the infinite path 
spaces, and a morphism $\pi$ between the Cuntz groupoids $G_{V}$ and $G_{W}$. Hence, the Cuntz algebra $O_{3}$ is isomorphic to the $C^{*}$-algebra of a Fell bundle over $G_{W}$. Note that for $x \in X_{W}$,

$$
K(x)=\left\{(y, 0, z) \in G_{V} \mid \varphi(y)=\varphi(y)=x\right\} .
$$

The fibers $C^{*}(K(x))$ of the Fell bundle are isomorphic to $M_{2^{n}}$, where $n$ is the number of 1's in $x$. For $n=\infty, M_{2 \infty}$ is the UHF-algebra of type $2^{\infty}$. It is interesting to note that these fibers are different.

EXAMPLE 4.4. For a graph $V$ with no sinks consider the collapsing map $\phi$ onto the graph $Z$ with one vertex and one loop. In this case $X_{Z}=\{*\}$ and $G_{Z} \cong Z$. The morphism $\pi$ is given by $\pi(x, k, y)=k$ and it induces the canonical Z-grading on the $C^{*}$-algebra $C^{*}(V)$.

Remark 4.5. Notice that the open map $\varphi: X_{V} \rightarrow X_{W}$ from Proposition 4.2 may be interpreted as a factor map between the topological Markov shifts $\left(X_{V}, \sigma\right)$ and $\left(X_{W}, \sigma\right)$. More generally, let $X, Y$ be locally compact spaces and let $\sigma: X \rightarrow X, \tau: Y \rightarrow Y$ be two local homeomorphisms. Assume that there is a continuous surjection $\varphi: X \rightarrow Y$ such that $\tau \circ \varphi=\varphi \circ \sigma$. In the language of dynamical systems, $(X, \sigma)$ is an extension of $(Y, \tau)$, or $(Y, \tau)$ is a factor of $(X, \sigma)$. If $\varphi$ is also open, it induces an open surjective groupoid morphism

$$
\pi: G(\sigma) \rightarrow G(\tau) \quad \text { given by } \quad \pi(x, k, y)=(\varphi(x), k, \varphi(y)),
$$

where $G(\sigma)=\left\{(x, m-n, y) \in X \times \mathbf{Z} \times X \mid \sigma^{m} x=\sigma^{n} y\right\}$ and $G(\tau)$ is defined in the same way. These groupoids are amenable by Proposition 2.4 in [25]. Moreover, $\pi$ is a groupoid fibration.

Example 4.6. Let $X=\{1,2\}^{\mathrm{N}} \times \mathrm{T}, Y=\{1,2\}^{\mathrm{N}}$ and let $\sigma: X \rightarrow X$ be given by

$$
\sigma\left(a_{1} a_{2} \cdots, z\right)= \begin{cases}\left(a_{2} a_{3} \cdots, z^{2}\right) & \text { if } a_{1}=1 \\ \left(a_{2} a_{3} \cdots, z^{3}\right) & \text { if } a_{1}=2 .\end{cases}
$$

Let $\tau: Y \rightarrow Y$ and $\varphi: X \rightarrow Y$ be given by $\tau\left(a_{1} a_{2} \cdots\right)=a_{2} a_{3} \cdots$ and $\varphi(a, z)=a$.

In this case the $C^{*}$-bundle over the unit space $G(\tau)^{0}=\{1,2\}^{\mathrm{N}}$ has fibers over $a \in\{1,2\}^{\mathrm{N}}$ isomorphic to Bunce-Deddens algebras of type $2^{n} 3^{m}$ where $n, m \in \mathrm{N} \cup\{\infty\}$ are the number of $1^{\prime} s$ and $2^{\prime} s$ in $a$, respectively.

REMARK 4.7. The notion of graph morphism with the path lifting property can be generalized to continuous graphs (see [9]). This gives a larger class of examples. 


\section{Abelian Fell bundles}

Definition 5.1. A Fell bundle over a groupoid $H$ is called abelian if $E_{u}$ is an abelian $C^{*}$-algebra for all $u \in H^{0}$.

Example 5.2. Consider $\pi: G \rightarrow H$ a groupoid covering (see Definition 3.2). Then the corresponding Fell bundle $E(\pi)$ is abelian, since $\pi^{-1}\left(H^{0}\right)=$ $G^{0}$. So in the notation of Theorem 3.4, $E_{u}=C_{0}\left(\pi^{-1}(u)\right)$ for all $u \in H^{0}$.

Coverings of groupoids are intimately related to groupoid actions on spaces. We will prove that every covering comes from such an action and vice versa. Other examples are related to some groupoid extensions. A Fell line bundle is an abelian Fell bundle (see Example 5.5). The main result in this section is a structure theorem for abelian Fell bundles that, loosely speaking, asserts that every such bundle arises from a "twisted" covering.

Recall (see [18]) that a groupoid $G$ is said to act (on the left) on a locally compact space $X$, if there are given a continuous, open surjection $\rho: X \rightarrow G^{0}$ and a continuous map

$$
G * X \rightarrow X, \quad \text { write } \quad(g, x) \mapsto g \cdot x,
$$

where $G * X=\{(g, x) \in G \times X \mid s(g)=\rho(x)\}$, that satisfy

i) $\rho(g \cdot x)=r(g), \forall(g, x) \in G * X$,

ii) $\left(g_{1}, x\right) \in G * X,\left(g_{2}, g_{1}\right) \in G^{2}$ implies $\left(g_{2} g_{1}, x\right),\left(g_{2}, g_{1} \cdot x\right) \in G * X$ and

$$
g_{2} \cdot\left(g_{1} \cdot x\right)=\left(g_{2} g_{1}\right) \cdot x,
$$

iii) $\rho(x) \cdot x=x, \forall x \in X$.

Note that the fibered product $G * X$ has a natural structure of groupoid, called the semi-direct product or action groupoid and denoted by $G \ltimes X$ (cf. [2]), where

$$
\begin{gathered}
(G \ltimes X)^{2}=\left\{\left(\left(g_{2}, x_{2}\right),\left(g_{1}, x_{1}\right)\right) \mid x_{2}=g_{1} \cdot x_{1}\right\} \\
\left(g_{2}, g_{1} \cdot x_{1}\right)\left(g_{1}, x_{1}\right)=\left(g_{2} g_{1}, x_{1}\right) \\
(g, x)^{-1}=\left(g^{-1}, g \cdot x\right) .
\end{gathered}
$$

Here the source and range maps are

$$
\begin{aligned}
& s(g, x)=\left(g^{-1} g, x\right)=(s(g), x)=(\rho(x), x), \\
& r(g, x)=\left(g g^{-1}, g \cdot x\right)=(r(g), g \cdot x)=(\rho(g \cdot x), g \cdot x),
\end{aligned}
$$

and the unit space may be identified with $X$. 
For $G$ an étale groupoid, consider the groupoid morphism $\pi: G \ltimes X \rightarrow$ $G, \quad \pi(g, x)=g$. Then ker $\pi$ may be identified with $X$, and $C_{r}^{*}(G \ltimes X)$ may be regarded as the $C^{*}$-algebra of an abelian Fell bundle over $G$ since ker $\pi=X$ is amenable and $E(\pi)_{u} \cong C_{0}\left(\rho^{-1}(u)\right)$ for all $u \in G^{0}$.

Proposition 5.3. Let $G$ be an étale groupoid acting on the locally compact space $X$. Then the morphism $\pi: G \ltimes X \rightarrow G, \pi(g, x)=g$ is a covering, and gives rise to an abelian Fell bundle over G. Moreover, every covering of étale groupoids is of this form. More precisely, given a covering map $\pi: G \rightarrow H$ of étale groupoids, there is a space $X$ and an action of $H$ on $X$ such that $G \cong H \ltimes X$.

Proof. Let $g \in G$ and let $x \in(G \ltimes X)^{0}=X$ such that $\pi(x)=s(g)$; then $(g, x) \in G \ltimes X$ satisfies $\pi(g, x)=g$ and $s(g, x)=x$, and is the unique such element in $G \ltimes X$. Hence, $\pi: G \ltimes X \rightarrow G$ is a covering.

Conversely, let $\pi: G \rightarrow H$ be a covering of étale groupoids. Set $X=G^{0}$ and $\rho=\pi^{0}: X \rightarrow H^{0}$. The map $\pi * s: G \rightarrow H \ltimes X$ is a continuous bijection (bijectivity follows from the definition of covering). To prove that $\pi * s$ is open, consider

$\mathcal{U}=\{U \subset G \mid U$ is an open bisection of $G$

such that $\pi(U)$ is an open bisection of $H$ \}.

Then $\mathscr{U}$ forms a basis for $G$, and if $U \in \mathscr{U}$, then $(\pi * s)(U)$ is open in $H \ltimes X$. Hence, $\pi * s$ is open and thus an isomorphism of locally compact groupoids.

See [10, Theorem 1.8] for an analogous result when $H$ is a group. The authors give conditions under which the groupoid $C^{*}$-algebra is Morita equivalent to a crossed product of an abelian $C^{*}$-algebra by an action of $H$.

ExAmple 5.4. Let $\pi: G \rightarrow H$ be an open surjective morphism where $G$ and $H$ are étale groupoids. Suppose that the restriction of $\pi$ to $G^{0}$ induces a homeomorphism $G^{0} \cong H^{0}$ and that $A=\pi^{-1}\left(H^{0}\right)$ is a sheaf of abelian groups over $G^{0}$. Then the resulting sequence:

$$
A \stackrel{\iota}{\longrightarrow} G \stackrel{\pi}{\longrightarrow} H
$$

is called an abelian extension where $\iota$ is the inclusion map. Since $A$ is amenable, the main theorem in $\S 3$ applies and we get a Fell bundle $E=E(\pi)$ over $H$. Moreover, $E_{u}=C^{*}\left(A_{u}\right)$ for all $u \in H^{0}$, where $A_{u}$ is the fiber of $A$ over $u$. Since $A$ is a sheaf of abelian groups, $E_{u}$ is abelian for all $u \in H^{0}$. Hence, $E$ is an abelian Fell bundle.

Example 5.5. Let $G$ be a proper T-groupoid over $H$, that is, $G$ is a groupoid endowed with the structure of a principal T-bundle over $H$ compatible with 
the groupoid structure (see [11]). Form the associated line bundle:

$$
E=G * \mathrm{C}=(G \times \mathrm{C}) / \mathrm{T}
$$

(where $t(g, z)=\left(t \cdot g, t^{-1} z\right)$ ). One defines multiplication and involution as follows:

$$
\begin{aligned}
\left(g_{1}, z_{1}\right)\left(g_{2}, z_{2}\right) & =\left(g_{1} g_{2}, z_{1} z_{2}\right), \\
(g, z)^{*} & =\left(g^{-1}, \bar{z}\right) .
\end{aligned}
$$

One verifies that $E$ is a Fell bundle over $H$ with these operations and that $E_{u} \cong \mathrm{C}$ for all $u \in H^{0}$. A bundle of this type is called a Fell line bundle. Any Fell bundle for which $E_{h}$ is one dimensional for all $h \in H$ is of this type. Its $C^{*}$-algebra is isomorphic to the twisted groupoid $C^{*}$-algebra of $G$ (see [20]).

THEOREM 5.6. Given a saturated abelian Fell bundle E over an étale groupoid $H$, there is a groupoid $G$, a covering $\pi: G \rightarrow H$ and a one-dimensional Fell bundle $L$ over $G$ such that $C_{r}^{*}(L) \cong C_{r}^{*}(E)$.

Proof. Set $X=\widehat{C_{0}\left(E^{0}\right)}$ i.e. $C_{0}\left(E^{0}\right)=C_{0}(X)$. Since $C_{0}(X)$ is the $C^{*}$ algebra of a bundle over $H^{0}$, we get a continuous open surjection $\rho: X \rightarrow H^{0}$ (see [15]). For $u \in H^{0}$ we have an isomorphism $j_{u}: E_{u} \cong C_{0}\left(X_{u}\right)$ where $X_{u}=\rho^{-1}(u)$; for each $h \in H, E_{h}$ is a $C_{0}\left(X_{r(h)}\right)-C_{0}\left(X_{s(h)}\right)$ equivalence bimodule and hence we get a homeomorphism $\alpha_{h}: X_{s(h)} \rightarrow X_{r(h)}$ (see [22, Corollary 3.33], [26, Corollary 6.27]). This defines a map $H * X \rightarrow$ $X,(h, x) \mapsto \alpha_{h}(x)$. We wish to show that this defines an action of $H$ on $X$ (see the definition following Example 5.2). Conditions i) and iii) are immediate, while condition ii) follows from the isomorphism $E_{h_{1} h_{2}} \cong E_{h_{1}} \otimes E_{h_{2}}$.

By Lemma 2.3, if $U$ is an open bisection of $H$, the completion of $C_{c}(U, E)$ may be endowed with the structure of an $A-B$ equivalence bimodule, where $A=C_{0}(r(U), E)$ and $B=C_{0}(s(U), E)$, with the natural inner products and actions. Note that $A \cong C_{0}\left(\rho^{-1}(r(U))\right)$ and $B \cong C_{0}\left(\rho^{-1}(s(U))\right)$. So again by [22, Corollary 3.33]) there is a homeomorphism $\rho^{-1}(s(U)) \cong \rho^{-1}(r(U))$ compatible with the above fiberwise homeomorphisms $\alpha_{h}$. This proves that the map $H * X \rightarrow X$ is continuous. Thus, $H$ acts on $X$. We set $G=H \ltimes X$ and by the above proposition, the map $\pi: H \ltimes X \rightarrow H$ given by $\pi(h, x)=h$ is a covering.

We construct $L$ piecewise as follows. Let $h \in H$; as noted above, $E_{h}$ is a $C_{0}\left(X_{r(h)}\right)-C_{0}\left(X_{s(h)}\right)$ equivalence bimodule and the equivalence induces a homeomorphism $\alpha_{h}: X_{s(h)} \rightarrow X_{r(h)}$. Then by Proposition A3 in [21] there is a Hermitian line bundle $L(h)$ over $\pi^{-1}(h)=\{(h, x) \mid \rho(x)=s(h)\}$ and an isomorphism $\iota_{h}: E_{h} \cong C_{0}\left(\pi^{-1}(h), L(h)\right)$ such that for all $\xi, \eta \in E_{h}$ and 
$g \in \pi^{-1}(h)$ we have

$$
\begin{aligned}
& j_{r(h)}\left(\xi \eta^{*}\right)(r(g))=\left\langle\iota_{h}(\eta)(g), \iota_{h}(\xi)(g)\right\rangle \\
& j_{s(h)}\left(\xi^{*} \eta\right)(s(g))=\left\langle\iota_{h}(\xi)(g), \iota_{h}(\eta)(g)\right\rangle,
\end{aligned}
$$

where $\langle\cdot, \cdot \cdot\rangle$ denotes the fiberwise sesquilinear inner-product (conjugate linear in the first variable). For $\left(h_{1}, h_{2}\right) \in H^{2}$ with $u=s\left(h_{1}\right)=r\left(h_{2}\right)$, we have an isomorphism $m: L\left(h_{1}\right) \otimes_{C_{0}\left(X_{u}\right)} L\left(h_{2}\right) \cong L\left(h_{1} h_{2}\right)$ such that for $\xi_{1} \in E_{h_{1}}$ and $\xi_{2} \in E_{h_{2}}$ we have

$$
m\left(\iota_{h_{1}}\left(\xi_{1}\right) \otimes \iota_{h_{2}}\left(\xi_{2}\right)\right)=\iota_{h_{1} h_{2}}\left(\xi_{1} \xi_{2}\right) .
$$

Moreover, involution on $E$ defines a conjugate linear isomorphism $L(h) \cong$ $L\left(h^{*}\right)$ for all $h \in H$. Now for $g \in G$, we define $L_{g}$ as the fiber over $g$ of the Hermitian line bundle $L(\pi(g))$ (note $L_{g} \cong \mathrm{C}$ ). We use the above operations to define multiplication and involution.

We wish to endow $L$ with the structure of a complex line bundle over $G$. Let $g \in G$, and let $U$ be an open bisection of $H$ containing $\pi(g)$. Then $C_{0}\left(\rho^{-1}(r(U))\right)$ and $C_{0}\left(\rho^{-1}(s(U))\right)$ are abelian and Morita equivalent (and hence isomorphic as noted above). Again by [21, Proposition A3] the equivalence bimodule may be identified with $C_{0}\left(\pi^{-1}(U), L^{U}\right)$ where $L^{U}$ is a Hermitian line bundle over $\pi^{-1}(U)$. One checks that $\left.L(h) \cong L^{U}\right|_{\pi^{-1}(h)}$ for $h \in U$. This gives us the topology of $L$ as a line bundle over $G$. The product and involution defined above are compatible with this topology and, hence, $L$ is a Fell bundle over $G$. It follows that $C_{r}^{*}(L) \cong C_{r}^{*}(E)$, where the map $\psi: C_{c}(L) \rightarrow C_{c}(E)$ is given by $\psi(f)(h)=\iota_{h}^{-1}\left(\left.f\right|_{\pi^{-1}(h)}\right)$.

Note that $E$ may be regarded as a push forward of $L$ under $\pi$. Specializing Example 5.4 to the case of groups, the above theorem yields a result that is no doubt well known to specialists.

Corollary 5.7. Let $\pi: G \rightarrow H$ be a surjective homomorphism, where $G$ and $H$ are discrete groups, such that $A=\operatorname{ker} \pi$ is abelian. Then we get an action of $H$ on the dual $\hat{A}$ and a Fell line bundle over the groupoid $H \ltimes \hat{A}$ defined by a two-cocycle $\omega:(H \ltimes \hat{A})^{2} \rightarrow \mathrm{T}$ such that $C_{r}^{*}(G) \cong C_{r}^{*}(H \ltimes \hat{A}, \omega)$.

Note that the line bundle over $H \rtimes \hat{A}$ is topologically trivial since we can construct cross-sections, but the cocycle is not necessarily a coboundary.

Example 5.8. For the first description of the Heisenberg group $G$ in Example 3.5 as an extension of $Z^{2}$ by its center $Z$, the action of $Z^{2}$ on $T \cong \hat{Z}$ is trivial, therefore the groupoid $Z^{2} \ltimes T$ is just the cartesian product $Z^{2} \times T$ 
with $(a, b, z)$ composable with $\left(a^{\prime}, b^{\prime}, z^{\prime}\right)$ iff $z=z^{\prime}$, and $(a, b, z) \cdot\left(a^{\prime}, b^{\prime}, z\right)=$ $\left(a+a^{\prime}, b+b^{\prime}, z\right)$. The cocycle $\omega:\left(\mathrm{Z}^{2} \ltimes \mathrm{T}\right)^{2} \rightarrow \mathrm{T}$ is given by

$$
\omega\left((a, b, z),\left(a^{\prime}, b^{\prime}, z\right)\right)=z^{a b^{\prime}} .
$$

Indeed, from the group operation in $G$, it follows that the map $f: \mathbf{Z}^{2} \times \mathbf{Z}^{2} \rightarrow \mathbf{Z}$ associated to the group extension is given by $f\left((a, b),\left(a^{\prime}, b^{\prime}\right)\right)=a b^{\prime}$, and since $Z \cong \hat{\mathrm{T}}$, we get the formula for $\omega$.

For the second description of $G$ as a semidirect product $Z^{2} \rtimes Z$, the cocycle is trivial since the extension splits, but the action of $\mathbf{Z}$ on $\widehat{Z}^{2} \cong \mathrm{T}^{2}$ is given by $\alpha(x, y)=(x, x y)$, and is induced by the conjugation with $[0,1,0]$ on $Z^{2}$ and dualization.

\section{REFERENCES}

1. Anderson, J., and Paschke, W., The rotation algebra, Houston J. Math. 15 no. 1 (1989), 1-26.

2. Anantharaman-Delaroche, C., and Renault, J., Amenable groupoids. With a foreword by Georges Skandalis and Appendix B by E. Germain, Monographies de L'Enseignement Mathématique, 36, L'Enseignement Mathématique, Genève, 2000.

3. Brown, L., Green, P., Rieffel, M. A., Stable isomorphism and strong Morita equivalence of $C^{*}$-algebras, Pacific J. Math. 71 no. 2 (1977), 349-363.

4. Brown, R., Danesh-Naruie, G., Hardy, J. P. L., Topological groupoids II. Covering morphisms and G-spaces, Math. Nachr. 74 (1976), 143-156.

5. Deaconu, V., Groupoids associated with endomorphisms, Trans. Amer. Math. Soc. 347 (1995), 1779-1786.

6. Fell, J. M. G., An extension of Mackey's method to Banach*-algebraic bundles, Mem. Amer. Math. Soc. 90 (1969).

7. Fell, J. M. G., Induced representations and Banach *-algebraic bundles, Lecture Notes in Math. 582 (1977).

8. Fell, J. M. G., and Doran, R. S., Representations of*-algebras, locally compact groups, and Banach *algebraic bundles, vol. 2, Academic Press (1988).

9. Katsura, T., A class of $C^{*}$-algebras generalizing both graph algebras and homeomorphism $C^{*}$-algebras. I. Fundamental results, Trans. Amer. Math. Soc. 356 no. 11 (2004), 42874322 .

10. Khoshkam, M., Skandalis, G., Regular representation of groupoid $C^{*}$-algebras and applications to inverse semigroups, J. Reine Angew. Math. 546 (2002), 47-72.

11. Kumjian, A., On equivariant sheaf cohomology and elementary $C^{*}$-bundles, J. Operator Theory 20 (1988), 207-240.

12. Kumjian, A., Fell bundles over groupoids, Proc. Amer. Math. Soc. 126 no. 4 (1998), 11151125.

13. Kumjian, A., Notes on $C^{*}$-algebras of graphs, Contemp. Math. 228 (1998), 189-200.

14. Landstad, M. B., Phillips, J., Raeburn, I., and Sutherland, C. E., Representations of crossed products by coactions and principal bundles, Trans. Amer. Math. Soc. 299 (1987), 747784.

15. Lee, R. Y., On the $C^{*}$-algebras of operator fields, Indiana Univ. Math. J. 25 no. 4 (1976), 303-314. 
16. Landsman, N. P., and Ramazan, B., Quantization of Poisson algebras associated to Lie algebroids, in Groupoids in analysis, geometry, and physics, Boulder, CO, 1999 (A. Ramsay and J. Renault, editors), 159-192, Contemp. Math. 282 (2001).

17. Muhly, P. S., Bundles over groupoids, in Groupoids in analysis, geometry, and physics, Boulder, CO, 1999 (A. Ramsay and J. Renault, editors), 67-82, Contemp. Math. 282 (2001).

18. Muhly, P. S., Renault, J., and Williams, D., Equivalence and isomorphism for groupoid $C^{*}$ algebras, J. Operator Theory 17 (1987), 3-22.

19. Muhly, P. S., Renault, J., and Williams, D., Continuous-trace groupoid $C^{*}$-algebras, III, Trans. Amer. Math. Soc. 348 no. 9 (1996), 3621-3641.

20. Muhly, P. S., and Williams, D., Continuous-trace groupoid $C^{*}$-algebras, II, Math. Scand. 70 (1992), 127-145.

21. Raeburn, I., On the Picard group of a continuous trace $C^{*}$-algebra, Trans. Amer. Math. Soc. 263 no. 1 (1981), 183-205.

22. Raeburn, I., and Williams, D., Morita equivalence and continuous-trace $C^{*}$-algebras, Math. Surveys Monogr. 60 (1998).

23. Ramazan, B., Quantification par Déformation des Variétés de Lie-Poisson, Ph.D. Thesis, University of Orléans, 1998.

24. Renault, J., A Groupoid Approach to $C^{*}$-algebras, Lecture Notes in Math. 793 (1980).

25. Renault, J., Cuntz-like algebras, Operator theoretical methods (Timişoara, 1998), 371-386, Theta Found., Bucharest, 2000.

26. Rieffel, M. A., Induced Representations of $C^{*}$-algebras, Adv. Math. 13 (1974), 176-257.

27. Yamagami, S., On primitive ideal spaces of $C^{*}$-algebras over certain locally compact groupoids, in Mappings of operator algebras (H. Araki and R. Kadison, editors), 199-204, Progr. Math. 84 (1990).

DEPARTMENT OF MATHEMATICS

UNIVERSITY OF NEVADA

RENO NV 89557-0084

USA

E-mail: vdeaconu@unr.edu, alex@unr.edu,ramazan@unr.edu 